\title{
Impact of packing density on primary drying rate
}

\author{
Anna Matejčíková, Pavol Rajniak \\ Department of Chemical and Biochemical Engineering, Institute of Chemical and Environmental Engineering, \\ Faculty of Chemical and Food Technology, Slovak University of Technology in Bratislava, \\ Radlinského 9, 81237 Bratislava, Slovakia \\ pavol.rajniak@stuba.sk
}

\begin{abstract}
This work aimed to determine the effect of packing density on the sublimation rate in a laboratory freeze dryer. Total amount of sublimed water was determined by gravimetric method while a total of seven experiments were performed under the same conditions (pressure, temperature, and drying time), at different configuration of the vials. The experiments confirmed that the higher the packing density, the lower the drying rate, and vice versa, the lower the packing density, the higher the drying rate. The effect of vials packing density was confirmed by experiments using a plastic rack, while the vials were separated and thus the packing density was lower. In this case, the drying rate was more homogeneous, which contributes to the higher quality of the final product.
\end{abstract}

Keywords: freeze-drying, gravimetric studies, lyophilization, packing density

\section{Introduction}

Freeze-drying, also known as lyophilization, is a commonly used process in the pharmaceutical, biomedical, and biotechnology industries. The main purpose of freeze-drying is preserving products and prolonging shelf-life stability while diminishing damages caused by sample drying. The process includes three main steps: (i) freezing of the solution to a frozen state when up to $95 \%$ of water is frozen, (ii) primary drying, during which frozen water is removed by sublimation, and (iii) secondary drying to remove unfrozen water by desorption (Tang, 2004).

Although lyophilization is a well-known process, some misconceptions still persist, especially with the lyophilization of larger molecules. One of the issues is product heterogeneity throughout the batch, but also among different batches even at the same operating conditions. This problem has become the subject of various studies, and different sources of heterogeneities have been identified such as (i) the stochastic nature of nucleation, (iii) the edge vial effect, (iii) vial geometry heterogeneities, and (iv) the impact of excipients. Due to the concavity of the vials bottom, the vials dimension varies from vials to vials leading to differences in heat and mass transfer. However, this is a production limitation which cannot be affected and therefore it is not discussed. Also, the effect of excipients on batch heterogeneity is not discussed as it is not the subject of this study (Assegehegn et al., 2018; Hibler et al., 2012).

The freezing stage is an important step and plays a critical role in product quality and appearance. Freezing conditions (such as cooling rate, nuclea- tion temperature, annealing, etc.) define the microstructure and affect both, drying rate and appearance of the final product. During the freezing step, nucleation occurs and due to its stochastic nature, nucleation temperature can vary by as much as $10{ }^{\circ} \mathrm{C}$ throughout the batch. Different nucleation temperatures lead to different crystal sizes and therefore, the quality of the final product varies (such as porous structure, product temperature and moisture content) (Assegehegn et al., 2018).

Also different position of vials is of importance. Vials situated along the periphery, termed as the edge vials, receive more heat compared to the central ones due to the heating from the walls. This phenomenon is denoted as edge vial effect. Also, it is important to note that edge vials are surrounded by a guard rail which also contributes to the edge vial effect (Rambhatla, 2003). In regard to the position of the vials, a critical factor is the degree of physical contact between the vials which is quantified by packing density and it is also a potential source of heterogeneity. The vial packing density was originally proposed by Placek (Placek, 2001). However, in this study, modified packing density expressed by the following equation is used:

$$
\varnothing=\frac{A_{\text {vials }}}{A_{\text {total }}}
$$

Where $A_{\text {total }}$ represents the observed circle area with a certain radius from the monitored vial's center $\left[\mathrm{m}^{2}\right]$, and $A_{\text {vials }}$ is the area occupied by vials in the observed area excluding the area of the monitored vial $\left[\mathrm{m}^{2}\right]$.

Vial configuration is closely related to packing density. In case of traditional close triangular pack- 
ing, the vials are very close to each other and most vials have very high packing density. But due to the maximum space utilization, productivity is the highest. Daller and co-workers studied the impact of the lack of physical contact between vials as well as with the rack. They confirmed that using a rack system heat transfer can be controlled minimizing the edge vials effect. However, packing density was not evaluated in their work (Daller et al., 2020).

\section{Materials and Methods}

\section{Equipment and Materials}

A laboratory freeze-dryer Edwards Supermodulyo 12K Freeze Dryer equipped with two shelves was used; while only the first shelf was used for the studies. All vials (20R) with the external diameter of $29 \mathrm{~mm}$ were filled with $5 \mathrm{~mL}$ of distilled water and stoppered with $18 \mathrm{~mm}$ diameter stoppers.

\section{Freeze-drying Procedure}

All experiments were performed under the same conditions. First, vials were sequentially numbered and positioned in different arrangements on a stainless-steel tray and surrounded by a stainlesssteel frame. During the experiment, the stainlesssteel tray was removed so the vials were in direct contact with the shelf. Vials were filled with $5 \mathrm{~mL}$ of deionized water. The amount of sublimed water was determined gravimetrically, i.e. as the difference between the weights of the vial filled with deionized water before and after the sublimation. Gravimetric measurements belong to the simplest and, at the same time, the only methods providing detailed representation of the behavior of single vial and variability within the batch. For sublimation studies, sublimation of only $30 \%$ of water is sufficient, otherwise, partial lack of thermal contact between ice and the vial wall may occur (Pikal et al., 1983). Sublimation tests were running as follows:

\section{Holding system}

Each experiment was performed using different vials configuration. Four experiments were performed without a holding system and three experiments were performed with a plastic stand as a holding system. For triangular packing, square packing, and packing with inactive vials, a traditional metal frame was used. For separated vials, three commercial plastic racks of $24.2 \mathrm{~cm} \times 12.5 \mathrm{~cm}$, with $6 \times 3$ bottomless holes of $3 \mathrm{~cm}$ diameter were used, while a fully loaded plastic rack held 54 vials (Fig. 1). The following vials configurations were used:

- Triangular packing. The first sublimation test was performed with a full packing (total 136 vials), while the vials were arranged in a close triangular configuration which is a traditional configuration in freeze-drying processes as it is the most efficient use of space.

- Square packing is an arrangement where the rows of spheres arranged in vertical and horizontal alignments form a square. Compared to triangular packing, the square full packing contains only 126 vials, so the overall process productivity decreases.

- Inactive rows. Vials are arranged in a close triangular packing; however, rows 7-10 are inactive.

Tab. 1. Freeze-drying cycle for sublimation studies.

\begin{tabular}{|c|c|c|c|c|c|c|}
\hline \multirow[b]{2}{*}{ Step } & \multicolumn{2}{|c|}{ Time } & \multicolumn{2}{|c|}{ Temperature $\left[{ }^{\circ} \mathrm{C}\right]$} & \multicolumn{2}{|c|}{ Vacuum [mbar] } \\
\hline & $\begin{array}{c}\text { Duration } \\
\text { [min] }\end{array}$ & $\begin{array}{c}\text { Total } \\
{[\mathrm{hh}: \mathrm{mm}]}\end{array}$ & Set & Reach & Condenser & Chamber \\
\hline Tempering & 30 & $00: 30$ & -5 & -5.0 & - & - \\
\hline Loading & 5 & $00: 35$ & -5 & -5.0 & - & - \\
\hline Freezing & 40 & 00:40 & -30 & -5.0 & - & - \\
\hline Freezing & 25 & 01:05 & -30 & -13.3 & - & - \\
\hline Freezing & 22 & $01: 27$ & -30 & -22.7 & - & - \\
\hline Freezing & 33 & 02:00 & -30 & -30.1 & - & - \\
\hline Freezing & 40 & $02: 40$ & -30 & -30.3 & - & - \\
\hline Evacuation & 30 & 03:10 & -30 & -30.3 & 0.8 & 0.8 \\
\hline Primary drying & 5 & $03: 15$ & -5 & -30.0 & 0.8 & 0.8 \\
\hline Primary drying & 25 & $03: 40$ & -5 & -10.9 & 1.0 & 1.0 \\
\hline Primary drying & 30 & $04: 10$ & -5 & -5.2 & 1.0 & 1.0 \\
\hline Primary drying & 240 & $08: 10$ & -5 & -4.8 & 0.9 & 0.9 \\
\hline Stoppering/Unloading & 10 & 08.20 & - & - & - & - \\
\hline
\end{tabular}

The ramp rate and the maximum allowable vacuum pressure were limited by the lyophilizer. 
Vials are filled with water but full-stoppered so the sublimation does not occur.

- Empty circle. Another, traditional triangular packing is used with two inactive vials' circles. Vials are filled with water but full-stoppered. Both circles contain one active vial in the middle. Therefore it is possible to observe the behavior of two isolated vials. The first isolated vial is surrounded by a single circle of inactive vials, and the second lonely vial is surrounded by a doubleinactive circle of vials.

- A plastic stand is a good tool to provide the same distance between the vials; however, productivity is relatively decreased. Using a plastic stand, three different arrangements of vials were used containing 54 vials, 27 vials, and 14 vials, respectively. To eliminate the radiation effect from the walls, the batch was surrounded by a polystyrene wall to ensure insulation.

\section{Packing density}

For each vial, two different packing density values were considered with respect to the size of the monitored area (Fig. 2). The first monitored area was a circle with the diameter twice that of a vial and the second monitored area was a circle with the diameter three times that of a vial, represented on Fig. 2 by vial 19 and 23, respectively. Circle segments were calculated using the AutoCAD.

For vial 19, packing density was calculated according to equation (1) as follows: the numerator expressing the area occupied by vials $A_{\text {vials }}$ (area of monitored vial number 19 not included) was calculated as the sum of the section marked in blue; the denominator represents the total monitored area, i.e. it is calculated as the area of a circle with the diameter of $5.8 \mathrm{~cm}$.

$$
\varnothing_{19}=\frac{A_{\text {vials }}}{A_{\text {total }}}=\frac{6 \times A_{\text {section }}}{\pi \times 2.9^{2}}
$$

Also, for vial 23, packing density was calculated according to equation (1) as follows: the numerator expressing the area occupied by vials $A_{\text {vials }}$ (area of monitored vial number 23 not included) was calculated as the sum of section and e full circle marked in blue; the denominator represents the total monitored area, i.e. it is calculated as the area of a circle with the diameter of $8.7 \mathrm{cmr}$.

$$
\varnothing_{23}=\frac{A_{\text {vials }}}{A_{\text {total }}}=\frac{6 \times A_{\text {section }}+6 \times \frac{\pi \times 2.9^{2}}{4}}{\frac{\pi \times 9 \times 2.9^{2}}{4}}
$$

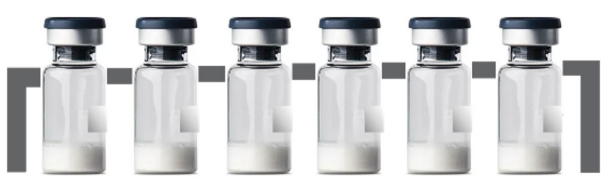

a)

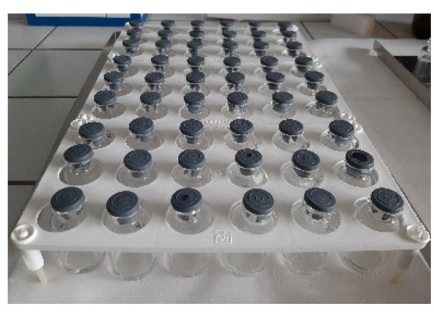

b)

Fig. 1. Plastic rack used for sublimation studies, a) cross section, b) top side.

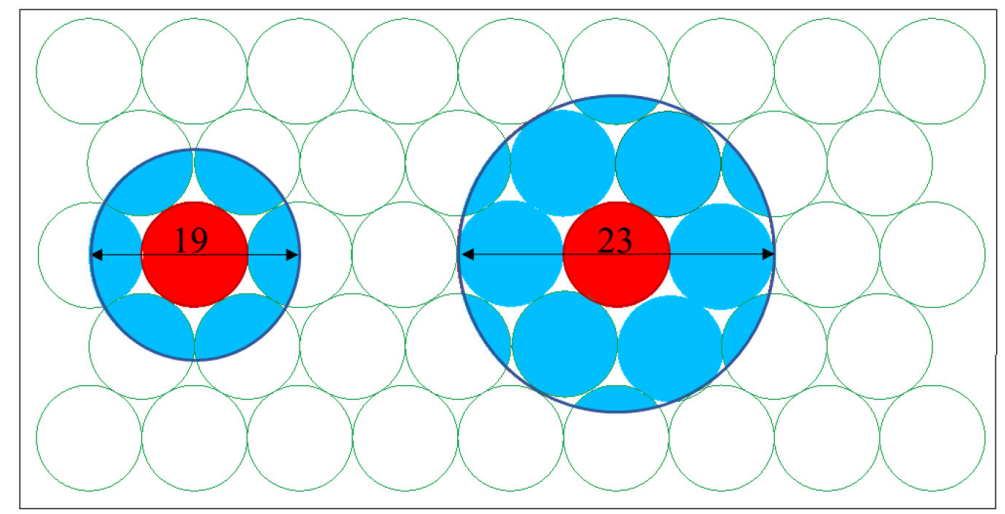

Fig. 2. Example of packing density calculation for vials marked in red, 19 and 23. In case of vial 19, the observed area is a circle with the diameter twice that of a vial. In case of vial 23, the observed area is a circle with the diameter three times larger than that of a vial. 


\section{Results and Discussion}

\section{Packing density evaluation}

Packing density was evaluated according to equation (1) for each experiment. AutoCAD was used to determine the area of the sections. The packing density is not the same for all vials but it depends on the vial position. For triangular packing, vials can be classified into five groups according to their position as follow (Pisano et al., 2011):

a) Edge vials located in the corner of the shelf (also known as hot vials, pink color),

b) Edge vials in contact with the rail (orange and yellow color),

c) Edge vials without contact with the rail (light green color),

d) Central vials (dark green color).

Each group is characterized by different amount of water sublimed due to the different heat transfer coefficient. Vials with the same packing density should show a comparable amount of sublimed water. However, it must be remarked that although the dark green vials are the same color, the amount of sublimed water may vary over a wider range. In dark green vials which are in direct contact with hot vials (periphery vials), the amount of mass sublimed may be higher compared to the central vials. It was experimentally confirmed that the lower the packing density, the higher the amount of sublimed water.

Packing density values together with the amount of water sublimed are listed in Tab. 2. In some measurements, so-called outliers occurred, i.e. either too low or too high values that are outside the expected trend. These deviations can be caused by either excessive or insufficient closure of the stopper. Another reason for too low values can be insufficient contact between the vial and the shelf. To avoid including erroneous measurements, five maxima and five minima were identified and eventual outliers were disregarded. From the results follows that using a square packing arrangement can easily homogenize the batch as there are only three different values of packing density. Using square packing looks like a good compromise for reducing heterogeneity within a batch due to its simplicity (no inactive vials are required) not significantly affecting the productivity. Obviously, the packing density for all vials is zero when using a plastic stand, which has a significant effect on the homogenization of the amount of sublimed water. However, the use of a stand to separate the vials is disadvantageous due to the low amount of the final product.

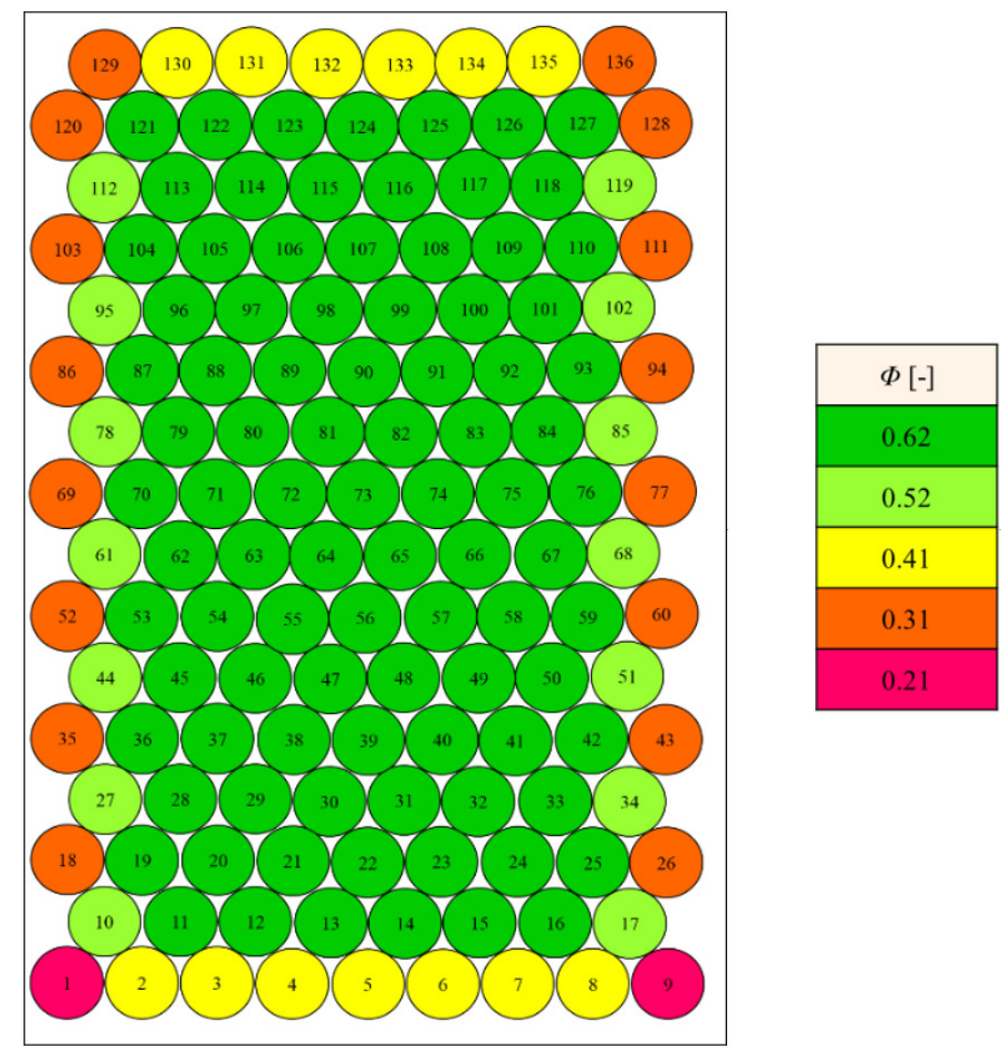

Fig. 3. Scheme of vial packing density classification as a function of their position in triangular packing. Values are different especially for the edge vials and central vials, which leads to different sublimation rates. 
Tab. 2. Evaluation of packing density for each vial configuration. The lowest $\left(\Delta m_{\min }\right)$ and the highest $\left(\Delta m_{\max }\right)$ amount of sublimed water were evaluated for each packing density.

\begin{tabular}{|c|c|c|}
\hline \multicolumn{3}{|c|}{ Packing denstiy 2D } \\
\hline$\Phi[\%]$ & $\Delta m_{\min }[\mathrm{g}]$ & $\Delta m_{\max }[\mathrm{g}]$ \\
\hline \multicolumn{3}{|c|}{ Triangular packing } \\
\hline 62.10 & 0.99 & 1.79 \\
\hline 51.75 & 1.18 & 1.78 \\
\hline 41.40 & 1.33 & 2.14 \\
\hline 31.05 & 1.56 & 2.05 \\
\hline 20.70 & 1.79 & 2.21 \\
\hline \multicolumn{3}{|c|}{ Square packing } \\
\hline 48.10 & 1.10 & 2.37 \\
\hline 35.21 & 1.26 & 2.11 \\
\hline 23.19 & 1.80 & 2.25 \\
\hline \multicolumn{3}{|c|}{ Triangular packing - empty circle } \\
\hline 62.10 & 0.98 & 1.77 \\
\hline 51.75 & 1.15 & 1.90 \\
\hline 41.40 & 1.17 & 2.31 \\
\hline 31.05 & 1.58 & 2.14 \\
\hline 20.70 & 1.70 & 2.20 \\
\hline 0.00 & 2.22 & 2.35 \\
\hline \multicolumn{3}{|c|}{ Triangular packing - empty rows } \\
\hline 62.10 & 1.74 & 2.31 \\
\hline 51.75 & 1.97 & 2.14 \\
\hline 41.40 & 2.00 & 2.59 \\
\hline 31.05 & 2.29 & 2.66 \\
\hline 20.70 & 2.61 & 2.77 \\
\hline \multicolumn{3}{|c|}{ Plastic stand -54 vials } \\
\hline 0.00 & 2.06 & 2.68 \\
\hline \multicolumn{3}{|c|}{ Plastic stand -27 vials } \\
\hline 0.00 & 2.68 & 3.13 \\
\hline \multicolumn{3}{|c|}{ Plastic stand -14 vials } \\
\hline 0.00 & 3.25 & 3.47 \\
\hline
\end{tabular}

Tab. 3. Comparison of the mass sublimed and homogeneity ratio in different configurations.

\begin{tabular}{lccccccc}
\hline & Triangular & \multirow{2}{*}{ Packing } & Square Packing & \multicolumn{3}{c}{ Empty } & \multicolumn{3}{c}{ Plastic Stand } \\
\cline { 4 - 8 } & & Circles & Rows & 54 vials & 27 vials & 14 vials \\
\hline$\Delta m_{\min }[\mathrm{g}]$ & 0.99 & 1.10 & 0.98 & 1.74 & 2.06 & 2.68 & 3.25 \\
$\Delta m_{\max }[\mathrm{g}]$ & 2.21 & 2.37 & 2.35 & 2.77 & 2.68 & 3.13 & 3.47 \\
$R$ & 2.23 & 2.15 & 2.41 & 1.59 & 1.30 & 1.17 & 1.07 \\
\hline
\end{tabular}

\section{Inhomogeneity ratio}

The amount of sublimed water is a good indicator of lyophilization inhomogeneity. To compare the heterogeneity of the batches, so-called inhomogeneity ratio expressed by the following equation is defined:

$$
R=\frac{\Delta m_{\max }}{\Delta m_{\min }}
$$

It follows from the above ratio that if the value approaches 1 , the batch is homogeneous, and, con- versely, the larger the ratio, the greater the difference between the minimum and maximum amount of sublimed water. The following table summarizes the values of the minimum and maximum amount of sublimed water as well as the inhomogeneity ratio for all measurements. The outliers were excluded, otherwise inhomogeneity ratio can be evaluated incorrectly (Tab. 3). The largest inhomogeneity ratio was determined for triangular packing. And vice-versa, the ratio approaches 1 if the packing density decreases, i.e. in experiments with plastic 
stands. When using plastic stands with 14 vials, the batch can be considered as homogeneous.

\section{Mapping of mass sublimed}

The amount of water sublimed was evaluated by the gravimetric method. In the beginning, vials were filled with $5 \mathrm{~mL}$ of pure water, weighted without stoppers $\left(m_{1}\right)$, and loaded into the drying chamber. This is followed by a freezing step and primary drying water sublimation according to the selected cycle. After approximately $30 \%$ of the ice was sublimed, the sublimation was stopped, vials were unloaded and reweighted $\left(m_{2}\right)$. The amount of sublimed water $(\Delta m)$ was calculated as a difference between $m_{1}$ and $m_{2}$.

Figs. 4 and 5 show the amount of sublimed water during individual experiments in color. In these experiments, a metal frame was used. The lowest

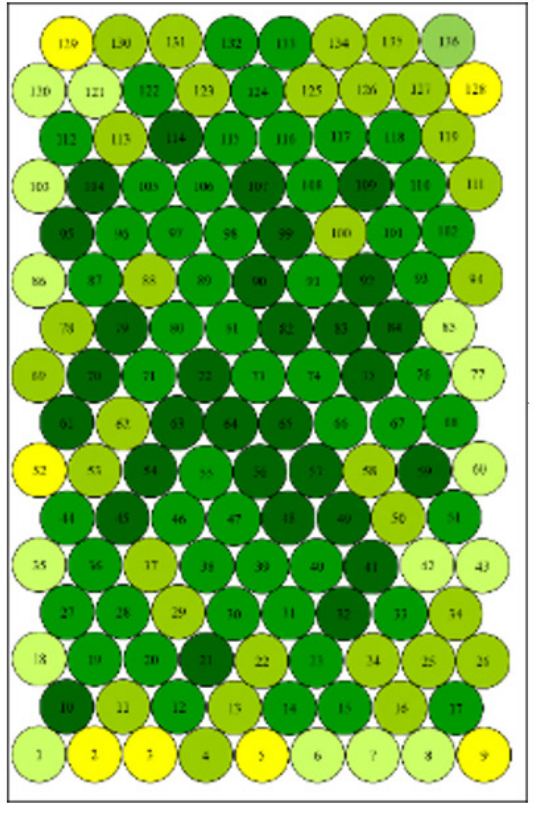

a)
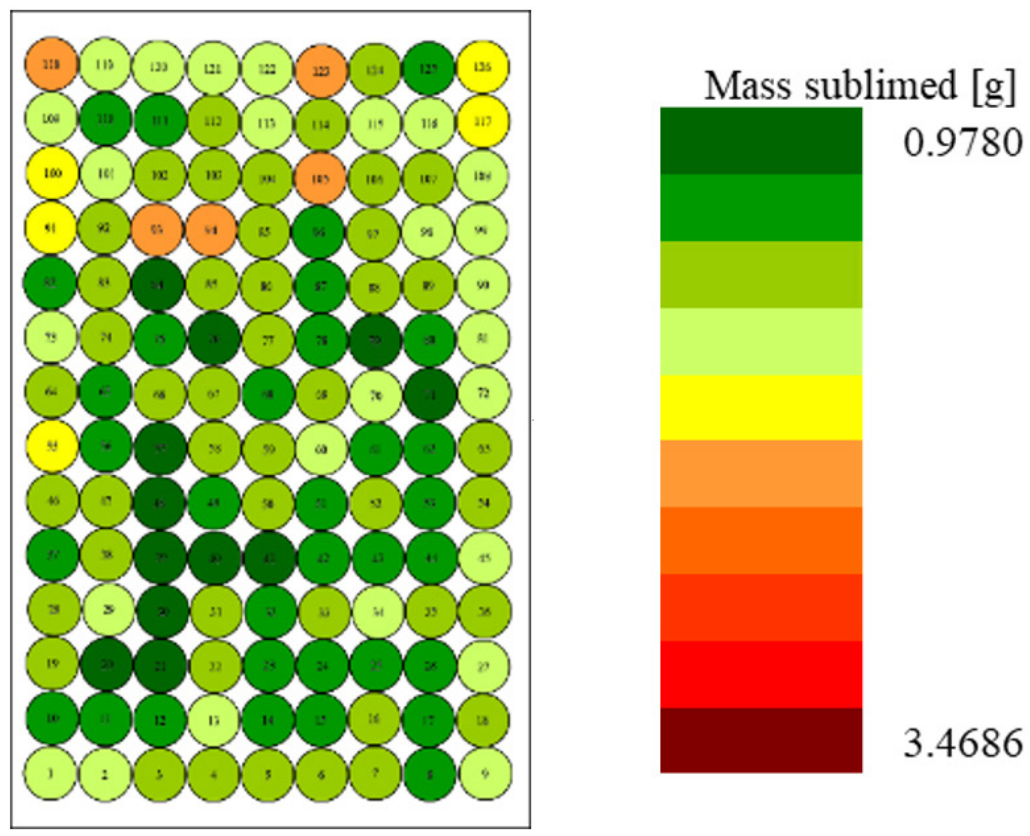

b)

Fig. 4. Effect of packing density with respect to vial arrangement. Two different full package configurations were used, (a) triangular packing (traditional approach), (b) square packing.

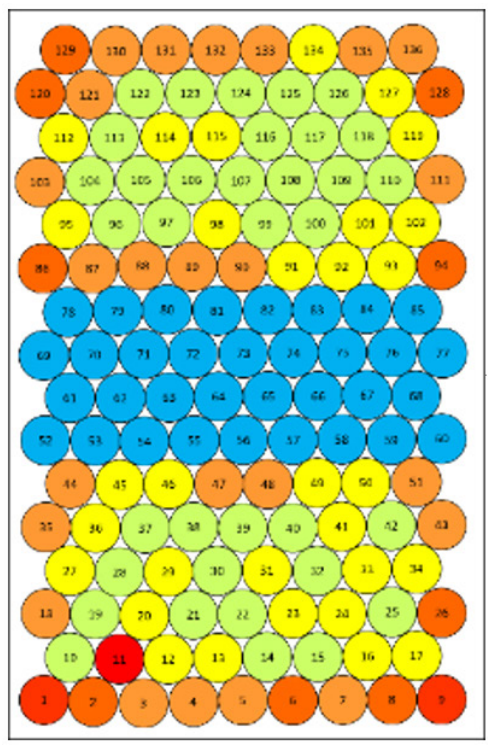

a)

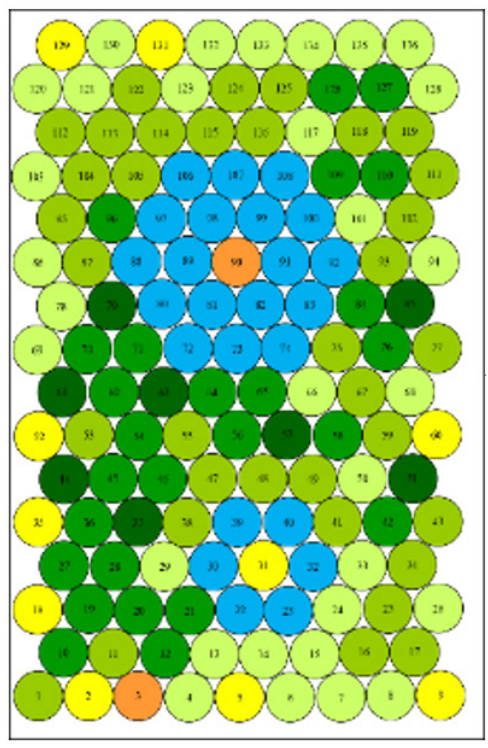

b)

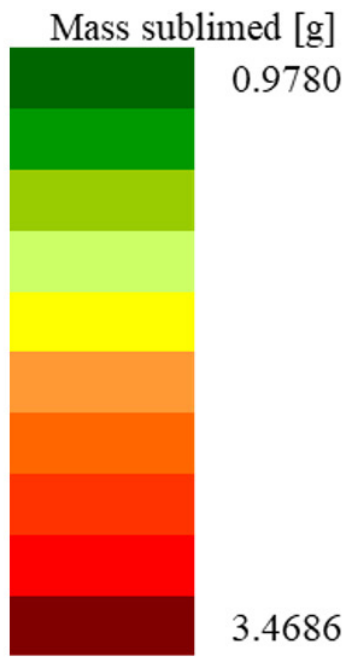

Fig. 5. Effect of empty vials in a traditional triangular packing and of packing density with respect to vial arrangement. The effect of empty rows and the effect of empty circles were monitored. 
amount of sublimed water was observed in vials marked with dark green color and, conversely, the highest amount of sublimed water was determined in vials marked with burgundy color. The blue vials were inactive and sublimation did not occur. When using a metal frame, the difference between the mass sublimed at periphery vs. central vials is significant. Mass sublimed in corner vial was as much as 2.23-fold higher compared to that in central vials. One possible explanation is that periphery vials are markedly heated from the walls and the primary drying rate is thus higher. Vice versa, the impact of walls is negligible in case of central vials due to the surrounding vials and sufficient distance from the walls.

Another explanation is the impact of packing density. Since the vials at the periphery have lower packing density and therefore lower competition, the primary drying rate is higher. In Figs. 4 and 5 it can be seen that in the vials located at the edge of the shelf, the amount of sublimed water is higher (marked from light green to orange-red) than in the middle of the shelf (dark green). It is also interesting to monitor the effect of inactive vials. In Fig. 5c), there are four rows of inactive vials that formally divide the batch into two parts. Since the vials located close to the inactive vials have no competitor, the packing density is lower and the sublimation is ultimately faster. Empty rows of vials are sometimes used in drugs manufacturing since they separate two different batches; however, these inactive vials cause inhomogeneity. Also, the impact of the empty circle was studied, Fig. $5 \mathrm{~d}$ ). In this case, it has been proved that even if the vial is in the middle of the shelf, it can be forced to behave like an edge vial just by surrounding it with inactive vials. It is obvious that if the vial has no competitors, i.e. low value of packing density, sublimation is faster. However, this arrangement was artificially created and is not practical to use.

To explain the impact of packing density, three experiments with a plastic rack were performed. Using a plastic rack, the vials are not in direct contact which results in higher sublimation rates.

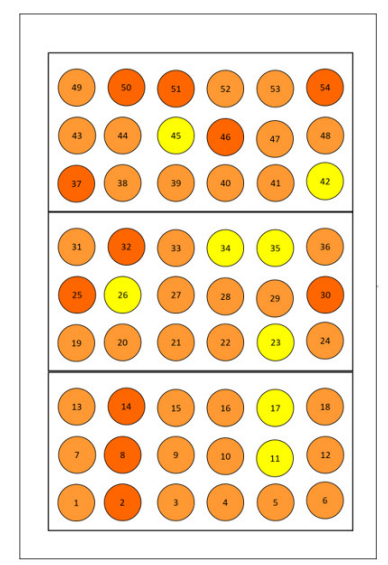

a)

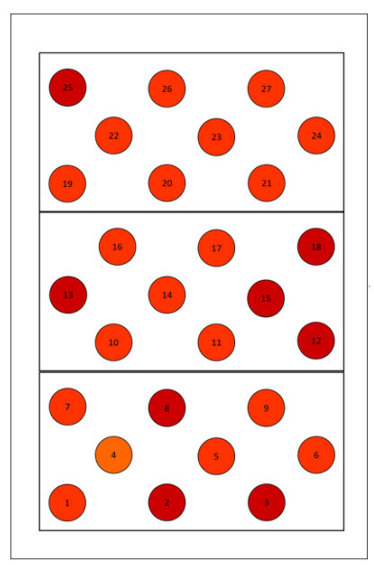

b)
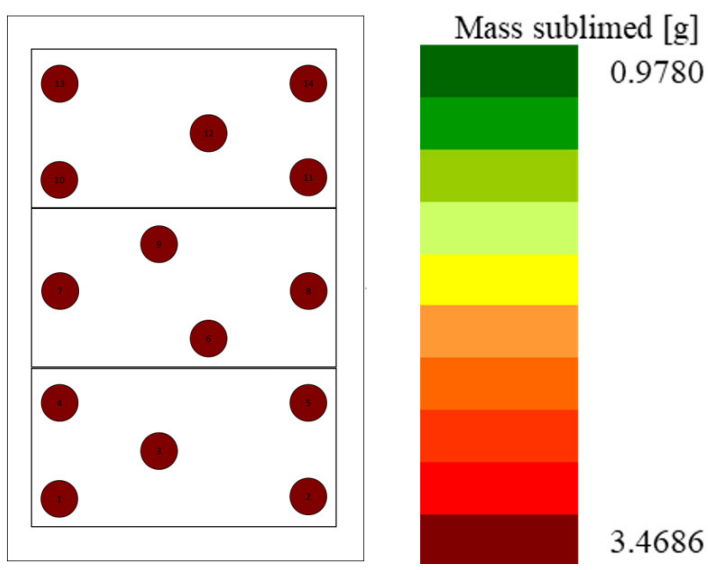

c)

Fig. 6. Comparison of total mass sublimed using plastic rack in three different configurations containing (a) 54 vials, (b) 27 vials, (c) 14 vials.

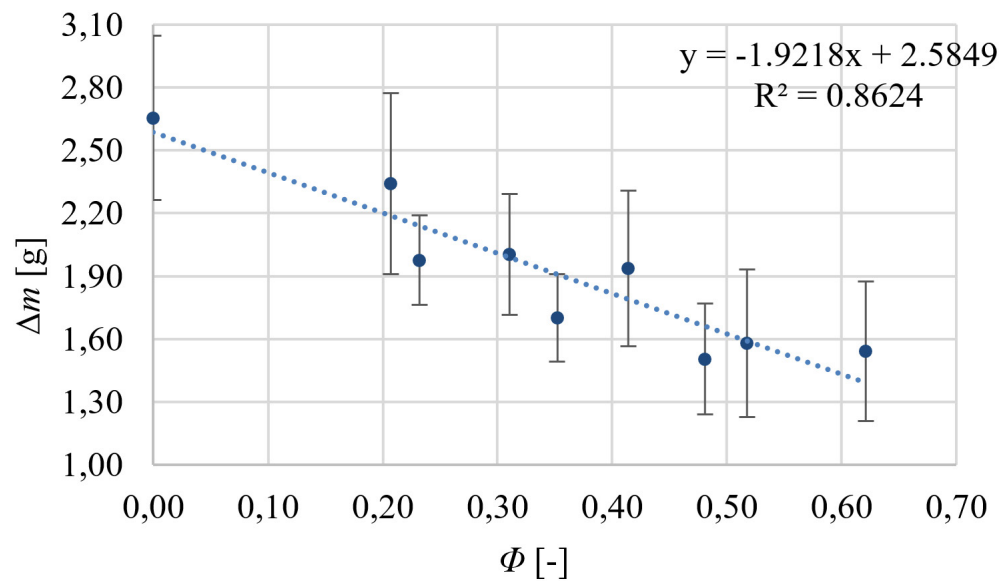

Fig. 7. Mass sublimed as a function of packing density including all measurements. 
Where the vials are separated, heat transfer is reduced to the shelf-to-vial transfer without the competition of neighboring vials. If the packing density is low enough, central vials also behave as vials at the edge. In comparison with full packing, mass sublimed in corner vial was only 1.07-fold higher compared to the central vials and this batch can thus be considered homogenous (Fig. 6 c). Finally, the effect of packing density on the amount of sublimed water is presented (Fig. 7). In this case, all measurements, including outliers, were taken into account. The results show that the highest rate of sublimation is reached at the packing density of 0 , i.e. if the vial is not surrounded by other active vials. However, this is disadvantageous in terms of productivity, which would drop sharply. The aim is not to ensure the highest rate of sublimation, but the highest homogeneity of the batch. It is possible to achieve better homogeneity by employing square packing or arranging vials with the packing density value in a narrow range for all vials by adding several empty vials.

\section{Conclusions}

Packing density is an important parameter significantly affecting freeze-drying heterogeneity. Batch heterogeneity is important considering product quality or process scale-up. In our study, the impact of packing density on the sublimation rate was evaluated with respect to the vials arrangement and position. The effect of packing density is summarized as follows: (i) the lower the packing density, the higher the sublimation rate. Results confirmed that the amount of sublimed water at the packing density of 0 is up to 2.04-fold higher than at 0.77 ; (ii) sublimation rate is higher in the edge vials (also termed as "hot" vials) compared to the central vials, the difference can be as much as 1.8-fold; (iii) the vials with the same packing density value (using a plastic rack) show similar values of sublimed water and can be considered homogeneous within the measurement error. Lyophilization is an energy and time-consuming process (i.e. also expensive) and forcibly reducing the packing density is not advantageous due to productivity decline. Therefore, it is reasonable to consider square packing of vials, which ensures an increase in the homogeneity of the batch with only a slight decrease in the productivity. Nowadays, not all causes of freeze-drying heterogeneity are fully known and understood and therefore the research on this issue will continue.

\section{Acknowledgment}

This research was supported by the Grant Scheme to support excellent teams of young researchers under the conditions of the Slovak University of Technology in Bratislava.

\section{References}

Assegehegn G, Fuente EB, Franco JM, Gallegos C (2018) The importance of understanding the freezing step and its impact on freeze-drying process performance. Journal of Pharmaceutical Science. 108: 1378-1395.

Daller S, Friess W, Schroeder R (2020) Energy transfer in vials nested in a rack system during lyophilization. Pharmaceutics 12.

Hibler S, Wagner Ch, Gieseler F (2012) Vial freeze-drying, Part 1: New insights into heat transfer characteristics of tubing and molded vials. Journal of Pharmaceutical Sciences. 101: 1189-1201.

Pikal MJ, Roy ML, Shah S (1983) Mass and heat transfer in vial freeze-drying of pharmaceuticals: Role of the vial. Journal of Pharmaceutical Sciences. 73: 1224-1237.

Pisano R, Fissore D, Barresi AA (2011) Heat transfer in Freeze-Drying Apparatus. Developments in Heat Transfer.

Placek J (2001) Heat transfer limitations, their reduction and control. Proceed. Freeze-Drying Pharm Biologic, Breckenridge.

Rambhatla S, Pikal MJ (2003) Heat and mass transfer scale-up issues during freeze-drying, I: Atypical radiation and the edge vial effect. AAPS PharmSciTech $4(2)$.

Tang X, Pikal MJ (2004) Design of freeze-drying processes for pharmaceuticals: practical advice. Pharm. Res. 21: 191-200. 